\title{
Desafíos y prácticas emergentes en la enseñanza del periodismo multimedia en Puerto Rico
}

\author{
Lourdes Lugo-Ortiz \\ Universidad de Puerto Rico. Escuela de Comunicación. Recinto de Río Piedras. \\ lourdeslugoortiz@gmail.com, lourdes.lugoortiz@upr.edu
}

Recibido: 17 de abril de 2017.

Aceptado: 19 de septiembre de 2017.

\begin{abstract}
Resumen
Las innovaciones en el ecosistema periodístico del siglo XXI han repercutido tanto en la práctica profesional como en los currículos académicos que forman a los futuros periodistas. Mediante el método de la encuesta y por primera vez en Puerto Rico, este estudio ausculta cómo las universidades han atendido las transformaciones del campo, qué prácticas emergentes han adoptado, qué retos enfrentan, con qué perfil docente cuentan y cómo ese perfil aporta a definir la cultura académica o profesional que prevalece en las instituciones educativas. La encuesta fue dirigida a los directores de todos los programas académicos y profesorado de periodismo del país. Mediante un análisis descriptivo, esta investigación encontró que los programas han adoptado un currículo multidisciplinario - estudian de forma separada el impreso, el audiovisual y el cibernético - y han transformado la mayoría de los cursos para incorporar el contenido multimedia. Las prácticas emergentes se reducen a la compra de equipo, aunque, para los encuestados, la falta de herramientas tecnológicas constituye un obstáculo en el proceso de enseñanza-aprendizaje, en consonancia con estudios en Latinoamérica. La facultad se inscribe en una cultura profesional: cuenta con una maestría como su grado terminal, con experiencia laboral y trabajos periodísticos más que investigativos. Aquellos con doctorado ostentan mayores haberes en investigación, labor creativa y experiencia profesional sobre aquellos con maestría; asimismo, las mujeres exhiben menos educación, experiencia en la enseñanza y ocupan, proporcionalmente, más puestos a tarea parcial que los hombres. El docente de periodismo, cónsono con el perfil profesional, se siente cómodo con las innovaciones tecnológicas y entiende que la formación multimedia no dificulta el proceso de enseñanza-aprendizaje. Los desafíos que enfrenta se relacionan con que el estudiantado no domina las destrezas fundamentales del periodismo (e.g., redacción, pensamiento crítico, etc.), más que con los cambios que ha traído el paradigma multimedia-digital.
\end{abstract}

Palabras clave: educación en periodismo, multimedia, Puerto Rico, cultura profesional, cultura académica, género. 


\title{
Challenges and emergent practices in the teaching of multimedia journalism in Puerto Rico
}

\begin{abstract}
Innovations in the 21 st century journalistic ecosystem have affected both professional practice and the academic curricula shaping future journalists. Through a survey method, this study, the first of its kind in Puerto Rico, looks at how universities have addressed transformations in the field, what emergent practices they have adopted, what challenges they face, what teacher profile makes up their faculties, and how this profile helps define the predominant academic or professional culture in educational institutions. The survey was sent to the directors of all academic and teaching programs dedicated to journalism throughout the country. Via a descriptive analysis, this research found that these programs have adopted a multidisciplinary approach - they study separately print, audiovisual, and cybernetic-, and have transformed most courses in order to incorporate multimedia content. Emergent practices are limited to the purchasing of equipment, although, according to respondents, the lack of technological equipment remains an obstacle in the teaching-learning process, an observation that coincides with other Latin American studies. The professors position themselves within a professional culture: they have a master's degree, work experience and journalistic work rather than research. Those with a doctorate degree have a firmer grasp of research, creative work, and professional experience than those with a master's; meanwhile, women exhibit less education and teaching experience, and do more part-time work than men. Journalism teachers, as befits their professional profile, are comfortable with technological innovations and understand that multimedia education does not make the teaching-learning process more difficult. The challenges they face have less to do with the transformations brought about by the multimedia-digital paradigm and more to do with the fact that students have still to master essential journalism skills (e.g., writing, critical thinking, etc.) Keywords: education in multimedia journalism, Puerto Rico, professional culture, academic culture, gender studies
\end{abstract}

\section{Desafios e práticas emergentes no ensino do jornalismo multimedia em Porto Rico}

\section{Resumo}

As inovações no ecossistema jornalístico do século XXI têm repercutido tanto na prática profissional quanto nos currículos acadêmicos que formam aos futuros jornalistas. Mediante o método do inquérito e pela primeira vez em Porto Rico, este estudo ausculta como as universidades tem atendido as transformações do campo, que práticas emergentes têm adotado, quais desafios enfrentam, qual o perfil docente e como esse perfil contribui na definição da cultura acadêmica ou profissional que prevalece nas instituições educativas. O inquérito foi dirigido para os diretores de todos os programas acadêmicos e ao professorado de jornalismo do país. Mediante uma análise descritiva, essa pesquisa encontrou que os programas tem adotado um currículo multidisciplinar - estudam de forma separada o impresso, o audiovisual e o cibernético- e tem transformado a maioria dos cursos para incorporar o conteúdo multimedia. As práticas emergentes se reduzem a compra de equipe, embora, para os entrevistados, a falta de ferramentas tecnológicas constituem um obstáculo no processo de ensino-aprendizagem, em consonância com estudos em Latino América. A faculdade se inscreve em uma cultura profissional: conta com um mestrado como seu grau terminal, com experiência laboral e trabalhos jornalísticos mais do quede pesquisa. Aqueles com doutorado ostentam maiores haveres em pesquisa, trabalho criativo e experiência profissional sobre aqueles com mestrado; além disso, as mulheres exibem 
menos educação, experiência no ensino e ocupam, proporcionalmente, mais postos de tarefas parciais que os homens. O docente de jornalismo, em concordância com o perfil profissional, se sente confortável com as inovações tecnológicas e entende que a formação multimedia não dificulta o processo de ensino-aprendizagem. Os desafios que enfrenta se relacionam com que os estudantes não dominam as destrezas fundamentais do jornalismo (e. g., redação, pensamento crítico, etc.), mais que com as mudanças que tem trazido o paradigma multimedia-digital.

Palavras chave: educação em jornalismo, multimedia, Porto Rico, cultura profissional, cultura acadêmica, gênero.

\section{Introducción}

Las transformaciones en el ecosistema periodístico del siglo XXI han repercutido tanto en la práctica profesional como en los programas que forman a los futuros periodistas. No es de extrañar que muchos centros educativos intenten dar cuenta de la producción hipertextual, multimedia, interactiva y multitemporal del ejercicio periodístico actual (Deuze, 2004, 2006; Royal, 2005; Salaverría, 2003, 2010 y 2011). Mas ¿cómo la academia ha acometido esta tarea?

Este estudio tiene el objetivo de explorar los desafíos que enfrentan las universidades ante el impacto de internet y las tecnologías digitales en la producción periodística del siglo XXI. En particular, ausculta, mediante el método de la encuesta y por primera vez en Puerto Rico, cómo los programas académicos han atendido las innovaciones del campo, qué prácticas emergentes han adoptado, qué retos enfrentan, con qué perfil docente cuentan los centros educativos y cómo este perfil aporta o no a la cultura académica y profesional institucional en tiempos multimedia.

\section{Algunas coordenadas sobre la educación en periodismo}

La educación universitaria ha sido el vehículo principal para la formación de periodistas en Puerto Rico, al igual que en otros países alrededor del mundo. Muchas otras naciones, sin embargo, descansan en las escuelas especializadas, en los mismos medios o en la combinación de algunas de estas modalidades. La estrategia de formación profesional de cada país responde a los propios contextos sociales, políticos, culturales y mediáticos, aunque, de forma acelerada, muchos más adoptan la educación universitaria o el sistema combinado de capacitación en la academia y las escuelas especializadas (Fröhlic \& Holtz-Bacha, 2003; Deuze, 2006). Estas transformaciones apuntan a un crecimiento en los niveles de "profesionalización, formalización y estandarización" del periodismo en el mundo entero (Deuze, 2006, p. 20).

La inauguración de la École Supérieure de Journalisme en Francia, a finales del siglo XIX (1895), marca el inicio de la formación educativa en periodismo (ESJ, s. f.). Sin embargo, la institucionalización de la enseñanza universitaria se originó en 1908 en Estados Unidos, en la Universidad de Misuri en Columbia (Bronstein \& Vaughn, 1998; 
Hansen, 2005; Hedges, 2006; Donsbach \& Fiedler, 2008). El ordenamiento del campo académico norteamericano - que surge en respuesta a unas necesidades de capacitación de las empresas mediáticas (Fuentes Navarro, 1992) - promovió la creación de escuelas alrededor del mundo, cuyos objetivos incluían la formación de periodistas.

La Universidad de Puerto Rico (UPR) en Río Piedras creó, en 1972, el primer programa académico en Comunicación en el país, uno de posgrado. Esa fundación ocurrió más de medio siglo después de que se iniciara la práctica del periodismo moderno, con el establecimiento del periódico El Mundo en 1919, y luego de casi cuatro décadas de que la Argentina instituyera el primer programa en periodismo en Latinoamérica (Fuentes Navarro, 1992; López García, 2010; Grisales, 2012). En 1975, la UPR inició la oferta de pregrado, cuya misión era la formación de profesionales en el área de la comunicación (Escuela de Comunicación, s. f.). A partir de entonces, se han creado unos veinte programas en la isla con el mismo fin (Escalante Rengifo, García de Jesús \& Oropeza Herrera, 2010), oferta que se inserta en los 1742 programas académicos latinoamericanos (FELAFACS-UNESCO, 2009).

\section{Ajustes al campo profesional}

El campo educativo en periodismo, tradicionalmente, ha tenido que acomodarse a las demandas del mercado, los públicos, así como a los cambios tecnológicos (Donsbach \& Fiedler, 2008). Mas, hoy día, enfrenta los desafíos de educar sobre prácticas que están en continua mutación y que se visibilizan en las maneras de producir la noticia, el flujo informativo, las relaciones con las audiencias, el negocio mediático, las estructuras organizacionales y la hibridación de las diferentes plataformas.

La dificultad que supone para la academia adaptarse a los cambios de la práctica periodística se suma a las críticas de las que históricamente ha sido objeto de parte del campo profesional y el académico. Se han puesto en tela de juicio los currículos, por ser muy teóricos o muy prácticos, o, simplemente, porque no enseñan las destrezas que requiere un periodista en un ecosistema multimedia-digital. Se cuestionan la capacidad y la experiencia del profesorado, así como algo mucho más básico: si un periodista debe estudiar periodismo o si debe recibir una formación amplia en diversas disciplinas, como economía o política (School of Journalism, 1987; Weaver \& Wilhoit, 1988; Fuentes Navarro, 1992; Reese, 1999; Dickson, 2000; Dickson \& Brandon, 2000a, 2000b; Deuze, 2004, 2006; Dates, 2006; Pierce \& Miller, 2007; Nolan, 2008; King, 2008; Hirst, 2010; Du \& Thornburg, 2011; Carnegie-Knight Initiative on the Future of Journalism, 2011; Mellado, 2011).

En respuesta a las presiones del campo profesional, desde finales del siglo XX y durante el siglo XXI, la academia en Estados Unidos, Europa, Latinoamérica y otras partes del mundo comenzó a impulsar cambios curriculares que fueran a la par con 
el nuevo ecosistema periodístico (Pierce \& Miller, 2007; Du \& Thornburg, 2011; Arroyo Cabello, 2011; Salaverría, 2011; Poynter Institute, 2013; García-Santamaría \& Barranquero Carretero, 2014; Lugo-Ortiz, 2016). Investigaciones con académicos y profesionales han identificado que las destrezas tradicionales (i. e., pensamiento crítico, redacción, ética, etc.) siguen siendo importantes en la formación del futuro periodista (Huang, Davison, Shreve, Davis, Bettendorf \& Nair, 2006), pero muchas otras, y en diversos grados, han señalado que conjuntamente hace falta adoptar una educación que dé cuenta del nuevo ecosistema digital y multimedia (Pierce \& Miller, 2007; Salaverría, 2010; Du \& Thornburg, 2011; Arroyo Cabello, 2011; Poynter Institute, 2013; García-Santamaría \& Barranquero Carretero, 2014).

La integración de lo multimedia-digital en los currículos ha seguido rutas diversas y disímiles que les han presentado grandes retos a los programas educativos. Un estudio comparativo entre Bélgica, Alemania y los Países Bajos evidenció que la educación en periodismo no avanza hacia la innovación, sino que sigue la práctica del campo, con una orientación técnica y profesional. Los cursos multimedia y convergentes en esos países se encuentran en la periferia (Deuze, Neuberger \& Paulussen, 2004). En España y para 2006, solo 7 por ciento de las materias de la licenciatura (lo que en Puerto Rico se denomina bachillerato) están vinculadas con el "ciberperiodismo" y suelen concentrarse en los últimos años de la carrera de estudio (Tejedor Calvo, 2006). No obstante, para 2011, Salaverría afirma que casi todos los programas en periodismo en ese país incorporaron cursos en medios digitales. En Estados Unidos, algunos programas han acogido cambios curriculares menores (i.e., en los cursos), mientras, en otros, las variaciones han sido radicales. Entre 1998 y 2002, el 60 por ciento de las escuelas de periodismo elaboraron cursos multimedia (Huang et al., 2006). Para 2003, un 85 por ciento de los principales programas estadounidenses adaptaron o comenzaron a adaptar sus currículos a la convergencia mediática, y, en un 23 por ciento, hicieron cambios mayores en sus mallas curriculares (Kraeplin \& Criado, 2005). No obstante, un 51 por ciento de los programas todavía estudian de forma separada el impreso, audiovisual y cibernético. En otras palabras, la investigación muestra que los programas que han incluido el aspecto convergente han adoptado un acercamiento multidisciplinario, visto como el nivel menor de integración de las disciplinas. La perspectiva interdisciplinaria, según Kraeplin y Criado (2005), representa el modelo más adecuado. Enseña al estudiantado las destrezas del impreso y del audiovisual en un contexto cibernético, medioambiente en el que se desenvolverán profesionalmente. Este acercamiento alienta el pensamiento holístico de la profesión (Kraeplin \& Criado, 2005). Coachie (2008), unos años después, evidenció que la mayoría de los programas en Estados Unidos han alterado sus currículos para adoptar lo multimedia. Solo un 9 por ciento reportó no haber iniciado una discusión al respecto. 
Lowrey, Daniels y Becker (2005), por su parte, definen las reformas curriculares en periodismo en tres etapas: estática, suplementaria y realineada. La estática se refiere a los programas que educan por medios específicos. La suplementaria, a los que continúan con los currículos tradicionales, pero los han complementado con cursos o secuencias multimedia, equivalentes al modo multidisciplinario, como lo identifican Kraeplin y Criado (2005). La realineada, a los que han reconceptualizado y han reamoldado las estructuras curriculares en respuesta a las presiones de la industria, a modo interdisciplinario. Lowrey et al. (2005) encontraron que la mayoría está experimentando con la convergencia, aunque solo un 31.7 por ciento de los programas son realineados, y un 52.6, suplementarios ${ }^{1}$.

Para Cuban (en Lowrey et al., 2005), no suelen ser exitosos los cambios curriculares que resultan de las presiones de los grupos externos, o de los sectores que determinan los recursos que recibirán las escuelas. En estos casos, las alteraciones suelen ser cosméticas y no estructurales, debido a que se posan sobre las perspectivas curriculares predominantes. Solo sirven, al final, para mostrar a los grupos externos que se está actuando. Según Lowrey et al. (2005), los programas que han intentado integrar la convergencia por las presiones externas han enfrentado la resistencia de los grupos internos (i. e., administradores, profesores y estudiantes). Generalmente, son departamentos o escuelas que se identifican con un área de especialidad particular, ostentan políticas internas limitantes, muestran dificultad en desmantelar los silos, y su facultad no cuenta con las destrezas requeridas. Sin embargo, los programas que se han movido hacia la convergencia han innovado en respuesta a las motivaciones internas para darle legitimidad a su malla curricular, y no por presiones externas. El interés de la facultad de crear un currículo convergente y los cambios en la industria son las variables determinantes para que el programa entrene a los estudiantes en diversas plataformas (Lowrey et al., 2005; Coachie, 2008).

El perfil del profesorado como dispositivo para que los programas adopten cambios curriculares también se identificó en el estudio de Coachie (2008), quien precisó que, a mayor cantidad de profesores innovadores y adaptadores tempranos, mayores probabilidades existen de que se impulsen cambios en los programas académicos y se adopten currículos convergentes. Coachie (2008) parte de la teoría de la difusión de las innovaciones de Roger, que explica los factores que influyen en la propagación de las nuevas ideas y tecnologías. El modelo identifica a los innovadores como aquellos

\footnotetext{
Daniels (en Castañeda, Murphy \& Hether, 2005) conceptualiza el estado de los currículos a base de tres modelos: convergentes, no convergentes y mixtos. El convergente desmantela las secuencias del impreso o televisivo y las sustituye por cursos multiplataformas; es lo que Kraeplin y Criado (2005) llaman el modelo interdisciplinario, y Lowrey et al., realineado. Los programas no convergentes dictan cursos multimedia, pero enseñan a los estudiantes en medios específicos y no enfatizan en el adiestramiento multiplataforma. Los mixtos ofrecen tanto el específico por medios como el multiplataforma. Estos últimos dos se clasificarían dentro de lo que Kraeplin y Criado (2005) identifican el acercamiento multidisciplinario, y Lowrey et al., suplementario.
} 
que son los primeros en adoptar ideas nuevas, manejan eficazmente la incertidumbre que trae la novedad y poseen la habilidad de maniobrar en situaciones tecnológicas complejas. Representan el 2.5 por ciento de la población. Les siguen los adoptadores tempranos (early adopters). Son líderes, modelos a seguir, respetados y exitosos; constituyen el 13.5 por ciento de la población. Los miembros de la mayoría temprana ocupan un lugar intermedio, interactúan con sus iguales, deliberan antes de someterse al cambio y no suelen ocupar posiciones de liderato; son el 34 por ciento de la población. Los de la mayoría tardía, otro 34 por ciento, adoptan la tecnología después de que mucha gente lo ha hecho, sienten la presión de sus iguales, son escépticos y cautelosos. Los rezagados usan el pasado como punto de referencia, están aislados y no tienen roles de liderato; componen el 16 por ciento de la población (Sahin, 2006; Coachie, 2008).

La disposición hacia la tecnología constituye una variable determinante que puede mediar en la adopción de los cambios curriculares en la enseñanza del periodismo. Muchos docentes nunca han trabajado en una sala multimedia, por lo que no han producido este tipo de contenido; carecen de una experiencia profesional dictada por las demandas del mundo digital o simplemente no pueden ser versados en todas las áreas (Auman \& Lillie, 2008; De Mendonça, Pereira \& Leal-Adghirni, 2012).

Mellado (2011) ha demostrado, además, que la cultura académica o profesional del docente contribuye a la profesionalización del periodista y a los estudios en periodismo. Mellado (2011) encontró que la prevalencia de una cultura docente sobre la otra - profesional o académica- moldea las que ostentan los departamentos o las escuelas que forman futuros periodistas. Por cultura académica o profesional se entiende la forma en que los educadores se distinguen en términos de sus características personales, experiencia profesional, dedicación a la enseñanza versus la investigación y producción científica. A la luz de ello, la cultura profesional de los docentes se define como aquella cuyo énfasis recae en la enseñanza y el trabajo ligado a los medios; mientras la académica se vincula con las investigaciones y publicaciones universitarias (Mellado, 2011).

Precisamente, estudios en diversos países, con mayor énfasis en Europa y Norteamérica, han delineado el perfil docente considerando la educación, producción investigativa, experiencia mediática y género, y han establecido correlaciones entre dicho perfil y el tipo de cultura que prevalece en las instituciones académicas. Investigaciones en Estados Unidos, Alemania y Australia identificaron que el profesorado de periodismo suele tener maestría o doctorado, distinto de Chile y México, cuya facultad cuenta mayormente con licenciatura (Fedler \& Counts, 1982; Weaver \& Wilhoit, 1988; Morton \& Beard, 2005; Rahkonen, 2007; Koivisto \& Thomas, 2007; FELAFACS-UNESCO, 2009; Mellado 2011). Si bien el perfil del profesorado en Chile es profesional, este se distancia del de Estados Unidos, en donde un profesor universi- 
tario de periodismo goza de un perfil académico: publica un libro y cuatro artículos en doce años de carrera. En Norteamérica, existe una correlación positiva entre productividad, programas doctorales y la facultad que posee estudios de posgrado (Weaver \& Wilhoit, 1988). Incluso, un estudio realizado por la Journalism School Administrators en Estados Unidos encontró que el profesorado con doctorado tiene experiencia profesional similar al que no exhibe tal grado (la diferencia es de menos de tres años): cuenta con, al menos, cinco años de experiencia y enseña los cursos teóricos (Stone, 1982). Asimismo, investigaciones en Estados Unidos han señalado que no existe una diferencia marcada entre el profesorado con doctorado y maestría, aunque el estudio de Weaver y Wilhoit (1988) apunta a que aquel con maestría tiene cerca de doce años de experiencia versus 6.5 años el que posee un doctorado.

Investigaciones producidas en Alemania y Australia identificaron que el género es una variable determinante en la productividad y experiencia mediática. Mientras en Australia los hombres tienen mayor productividad investigativa, en Alemania es a la inversa: las mujeres ostentan mayor productividad académica. Sin embargo, pesquisas en Estados Unidos no suelen encontrar grandes diferencias por razón de género en esas dos categorías (Fedler, Counts \& Smith, 1984; Mellado, 2011).

Pese a que ninguno de los estudios mencionados sobre el perfil de la facultad en Europa, Latinoamérica y Estados Unidos se vinculan con la integración de lo digital-multimedia en los currículos, las investigaciones sugieren que existe una relación entre la cultura docente y el contenido y orientación de los programas académicos. En ese sentido, entender el tipo de perfil que ostenta el docente en Puerto Rico contribuirá a identificar otro factor que incide en la cultura institucional que ostentan o adopten los centros educativos que forman a los futuros periodistas.

El éxito de la integración de las perspectivas multimedia en los programas académicos reside, conjuntamente, en la adopción de las nuevas estrategias de enseñanza, la contratación de docentes especializados y el adiestramiento de la facultad. Según Davis, la educación multimedia debe ir de la mano con el team teaching o enseñanza en equipo, dos o más docentes con cierto nivel de colaboración en la planificación y cátedra de un curso (En Auman \& Lillie, 2008, p. 361). No obstante, la eficacia de esta técnica depende del año curricular en la que se incorpore. Auman y Lillie (2008) hallaron que los estudiantes avanzados se benefician más de esta estrategia que los que están comenzando su carrera académica. Igualmente, las escuelas tendrán que depender de los profesores por contrato para suplementar su oferta docente (South, Nicholson \& Fisher, 2002 en Coachie, 2008, p. 9), así como de la reeducación de plantel docente regular. El conocimiento especializado por disciplina de los docentes serviría para que el profesorado se eduque entre sí, a modo de adiestramiento cruzado (cross-training). Los del impreso aprenderían de los del audiovisual, y viceversa (Kraeplin \& Criado, 2005). 
La capacitación externa es otro recurso, aunque Coachie (2008) encontró que la mayoría de los programas en Estados Unidos no entrenan a su facultad, debido a los costos, las limitaciones presupuestarias, la resistencia de los docentes y de los estudiantes.

La educación en periodismo enfrenta múltiples desafíos; resulta importante entender cómo la academia en Puerto Rico los ha manejado. Es decir, si la práctica profesional ha cambiado, ¿ los estudios en periodismo y el profesorado se han ajustado a esas transformaciones? Con este interés, este artículo explora respuestas a las siguientes preguntas: ¿qué innovaciones curriculares han incorporado los programas de periodismo?, ¿qué retos enfrenta la enseñanza de dicho campo profesional?, ¿qué tipo de educación continua ha adoptado el profesorado de periodismo para responder a las evoluciones de la industria?, ¿cuál es la disposición de los docentes hacia la tecnología, qué perfil ostentan y a qué cultura, profesional o académica, se orientan?

\section{Método}

Con una población de 3,5 millones de habitantes, Puerto Rico - un archipiélago caribeño- cuenta con 140 unidades académicas de educación superior, de las cuales veinte poseen programas que ofrecen desde certificaciones técnicas hasta maestrías en algún área de la Comunicación (M. Rivera Molina, Consejo de Educación de Puerto Rico, comunicación personal, 1 de diciembre de 2016). Sin embargo, solo once universidades incorporan cursos en periodismo o clases que integran algún elemento de esta área de estudio ${ }^{2}$.Tres de ellas son públicas, y ocho, privadas. Dos de los tres programas en universidades públicas - el de la Escuela de Comunicación de la Universidad de Puerto Rico Recinto de Río Piedras y el del Departamento de Comunicación TeleRadial de la Universidad de Puerto Rico Recinto de Arecibo- están acreditados, de forma voluntaria, por la Accrediting Council on Education in Journalism and Mass Communications (ACEJMC, 2016).

Los directores de los once programas educativos objeto de estudio facilitaron una lista de los docentes a tarea parcial y completa que imparten los cursos que incorporan la enseñanza del periodismo. Tanto a los directores como al profesorado se les envió, vía correo electrónico, una carta con el enlace a la encuesta. El instrumento, producido en una plataforma digital, contó con 41 preguntas para los profesores y 52 para los directores, aunque solo algunas de ellas sirven de base para la redacción de este artículo. Se sondearon las opiniones de los directores, porque están a cargo de delinear el rumbo curricular y administrativo de los programas, y las del profesorado a tiempo

\footnotetext{
Las once universidades que participaron en el estudio son las siguientes: American University; Pontificia Universidad Católica de Puerto Rico en Ponce; Universidad del Este; Universidad Interamericana, recintos de Bayamón y Ponce; Universidad Metropolitana; Universidad de Puerto Rico, recintos de Arecibo, Carolina y Río Piedras; Universidad del Sagrado Corazón y Universidad del Turabo.
} 
parcial y a tiempo completo, porque es el que imparte el conocimiento que contribuye a formar el futuro periodista. Las preguntas sobre el programa fueron dirigidas a los directores y profesores; aquellas relacionadas con la enseñanza del periodismo, solo a los docentes de esta disciplina. Esta pesquisa es la primera que se produce sobre este tema en Puerto Rico, pese a que estudios como el propuesto han proliferado en otros países, como Brasil, Chile y Estados Unidos (Singer, Craig, Allen, Whitehouse, Dimitrova \& Sanders,1996; Huang et al., 2006; Mellado, 2011; De Mendonça et al., 2012, y Boers, Ercan, Rinsdorf \& Vaagan, 2012). Como métodos complementarios, se estudiaron las plataformas digitales de los departamentos o escuelas y se obtuvo información contextual mediante los directores de cada programa.

Treinta y nueve (39) profesores enseñan cursos de periodismo o cursos que incorporan algún aspecto de esta disciplina; veintisiete (27) de ellos contestaron la encuesta, con un 69.23 por ciento de participación. La mayoría de los encuestados (78.57\%) labora en universidades privadas, porcentaje que guarda relación con la distribución de universidades privadas y recintos públicos: ocho privadas $(72.72 \%)$ y tres públicas ${ }^{3}$. Siete de los once directores de los programas participaron en la encuesta; seis de ellos trabajan en universidades privadas y uno, en un recinto de la pública ${ }^{4}$.

La mayoría de los once programas que cuentan con cursos de contenido periodístico están ubicados en el área metropolitana (72.73\%), en donde se concentra la mayor población del país. De las once universidades, solo dos ofrecen grados en periodismo a nivel de pregrado (bachillerato) y posgrado (graduado): la Universidad de Puerto Rico Recinto de Río Piedras, una licenciatura (bachillerato) en Comunicación con concentración en periodismo y una maestría en esta última área, y la Universidad del Sagrado Corazón, una concentración al nivel de pregrado, certificados de poslicenciaturas (o lo que se denomina posbachillerato) en periodismo y una maestría en Periodismo Digital. La primera es pública; la segunda, privada. El resto de las instituciones que incorporan cursos de periodismo ofrecen grados terminales en comunicación o algún área de la comunicación. Ninguna de ellas ofrece un grado doctoral en el campo.

\section{Hallazgos y análisis}

Muchos de los programas de Comunicación en Puerto Rico han atemperado sus cursos al mundo digital-multimedia. En otras palabras, han integrado la enseñanza del

\footnotetext{
3 La Universidad de Puerto Rico es la única institución de educación superior pública en el país. Su sistema se compone de once recintos, tres de ellos participaron en este estudio.

4 Al aplicar el factor de corrección para una población finita (CPF) en el muestreo del profesorado y de los directores, el error estándar se aproximó a cero, dado que el tamaño de la muestra (n) se acercó al tamaño de la población (N). Este cálculo tuvo el efecto de eliminar el margen de error por completo. Cuando $\mathrm{N}=\mathrm{n}$, la muestra se convierte en un censo; el error de muestreo resulta irrelevante, así como interpretar el valor p (Figueiredo Filho, Paranhos, Rocha, Batista, Silva Jr., Santos \& Marino, 2013).
} 
periodismo en plataformas múltiples, tomando en cuenta el contexto cibernético y la producción hipertextual y multitemporal (o que combina fácilmente las informaciones más recientes con las de archivo) de la práctica profesional actual (Deuze, 2004, 2006; Royal, 2005; Kraeplin \& Criado, 2005; Salaverría, 2003, 2010 y 2011). Tres cuartas partes enseñan el periodismo tanto de forma multimedia como por áreas individualizadas, y un 12.5 por ciento, de forma multimedia. Los currículos del país tienden a ser multidisciplinarios, suplementarios o mixtos y han ido incorporando los "recursos y las lógicas digitales" en la enseñanza (Salaverría, 2010, p. 248).

La integración de lo multimedia en los programas fue el resultado, en un 50 por ciento de las veces, de revisiones curriculares, y, en un 37.5 por ciento, fue concebida así desde sus inicios. Las revisiones para incorporar el elemento multimedia han sido significativas: el 37.50 por ciento de las escuelas o departamentos han cambiado todos los cursos; el 25 por ciento, más de la mitad de los cursos; el 12.50 por ciento, la mitad de los cursos, y el 25 por ciento no han variado los cursos (véase fig. 1). Las transformaciones en la industria constituyeron la razón principal para integrar lo multimedia (87.50\%); mientras una minoría de los programas (12.50\%) atribuyó los cambios a las sugerencias de alguna junta universitaria.

Figura 1. Cambios curriculares en escuelas o departamentos para integrar aspectos multimedia según los directores de los programas académicos*.

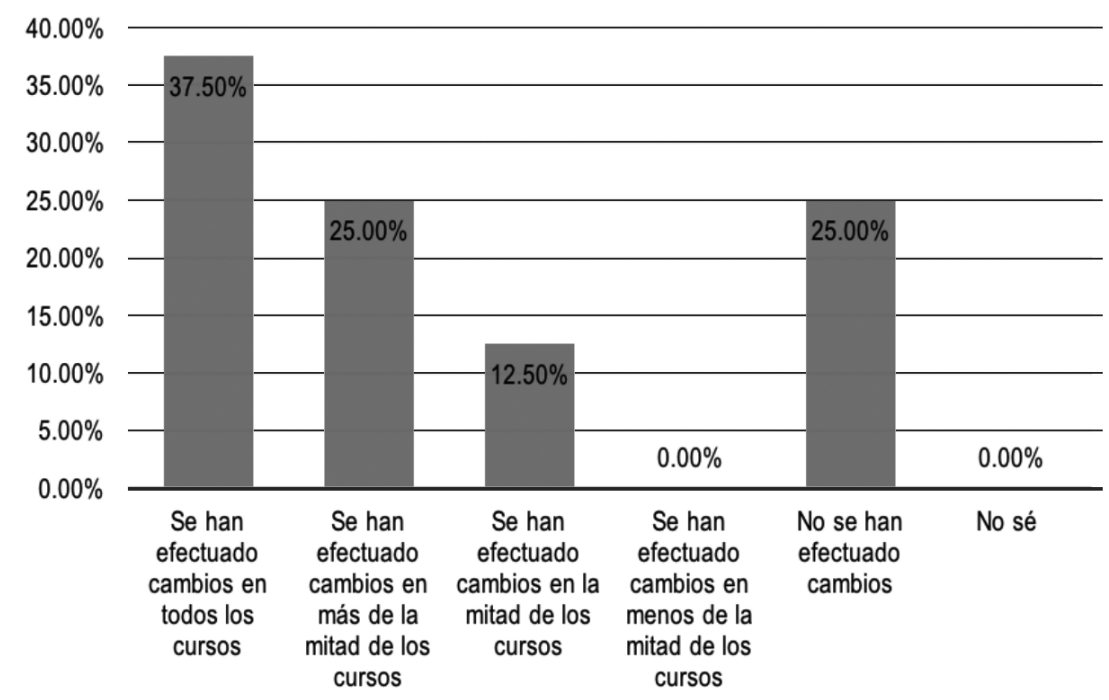

*Todos los datos de gráficas y tablas proceden de fuente propia. 
Como parte de la enseñanza multimedia, las escuelas o departamentos han comprado equipos o programas computadorizados (35.29\%). También, con mucho menor énfasis, han incorporado la enseñanza en equipo (17.65\%), creado salones multimedia (17.65\%), contratado profesores especialistas en el área de convergencia (11.76\%) y adiestrado a los docentes (5.88\%). (Véase fig. 2).

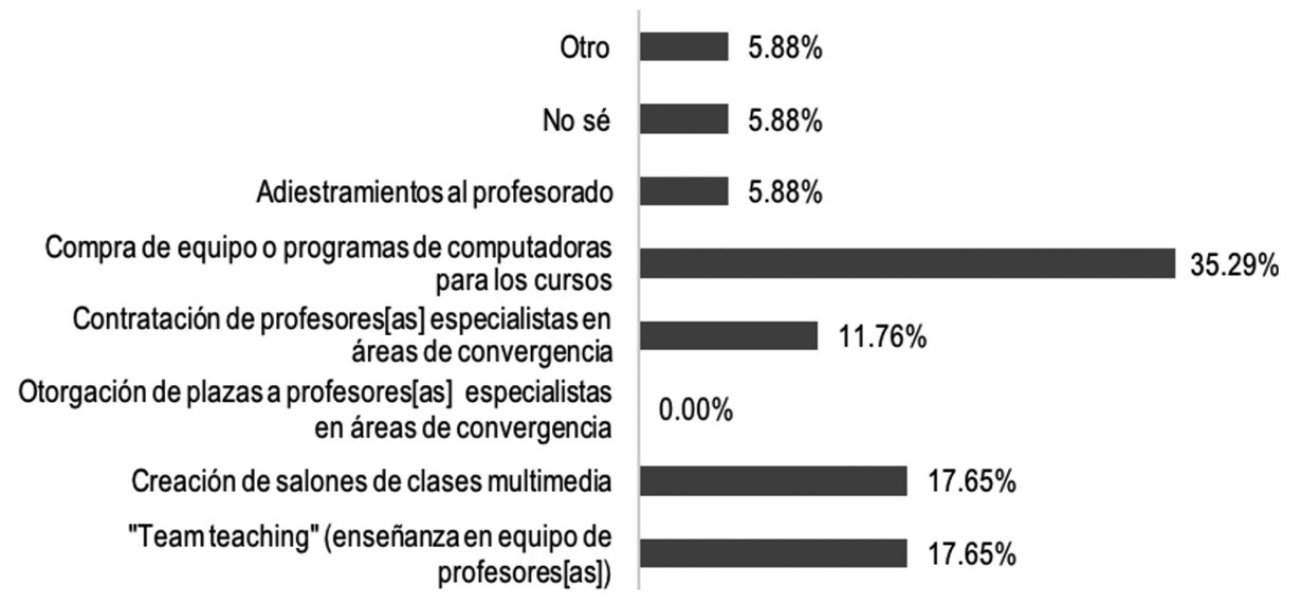

Figura 2. Cambios incorporados en los programas para adelantar la enseñanza multimedia de acuerdo con el profesorado de periodismo.

\section{Retos de la educación multimedia}

Los directores de los departamentos en Comunicación y el profesorado de periodismo coincidieron en que la estructura curricular (directores, 100\%; profesores, 60.71\%) y las limitaciones en la cantidad de horas créditos (directores, 100\%; profesores, 53.57\%) representan los principales obstáculos para que el estudiantado reciba una formación multimedia. Para los directores, además, constituyen trabas las diferentes perspectivas de la facultad sobre lo que es la educación en periodismo (80\%), la ausencia de experiencia profesional de los docentes $(60 \%)$ y la falta de tiempo o voluntad del profesorado para desarrollar cursos multimedia (60\%). Para la facultad, la burocracia administrativa también configura un impedimento importante (57.14\%). Aunque los directores y los docentes concordaron en varias de las dificultades, aquellas relacionadas con la gestión administrativa cobraron menor importancia para los directores, y aquellas que responsabilizan al profesorado tuvieron menor relevancia para estos últimos (véase tabla 1). 
Tabla 1. Retos principales que enfrenta la educación en periodismo multimedia de acuerdo con los directores de los programas y el profesorado.

\begin{tabular}{lcc}
\hline Reto & Directores \% & Profesores \% \\
\hline Estructura curricular & 100 & 60.71 \\
Limitaciones en la cantidad de horas créditos que el estudiante puede tomar & 100 & 53.57 \\
Diferencias entre la facultad sobre lo que es la educación en periodismo & 80 & 21.14 \\
Falta de experiencia profesional o conocimiento de la facultad & 60 & 28.57 \\
Falta de tiempo o voluntad para desarrollar cursos multimedia & 60 & 17.85 \\
Burocracia administrativa & 40 & 57.14 \\
Falta de cooperación de la facultad & 40 & 14.28 \\
\hline
\end{tabular}

Los desafíos que la facultad ha enfrentado en la enseñanza en los últimos diez años se vinculan principalmente con la falta de destrezas que exhibe el estudiantado más que con la integración de lo multimedia en el currículo. En una pregunta abierta, el 59.26 por ciento de los docentes identificaron que los retos principales que han encarado se relacionan con las deficiencias estudiantiles asociadas con las destrezas tradicionales del periodismo, y no con la incorporación de lo digital-multimedia en el salón de clases. Un 25.93 por ciento señaló que los desafíos están asociados solo a la integración de la enseñanza multimedia-digital, y un 14.81 por ciento, tanto a la falta de destrezas tradicionales de los alumnos como a la enseñanza del periodismo multimedia (véase fig. 3). Las carencias de las destrezas fundamentales del estudiantado que el profesorado mencionó son las siguientes: lenguaje oral y escrito; pensamiento crítico, lectura e investigación; e información, cultura y conocimiento general. Dentro de los retos vinculados con la enseñanza del periodismo multimedia, los docentes identificaron el desconocimiento del paradigma digital por parte de alumnos y del personal docente; el énfasis en lo tecnológico sobre los fundamentos del campo; el ajuste a las tecnologías digitales; los altos costos tecnológicos; la falta de equipo, instalaciones y laboratorios; y las estructuras universitarias rígidas que obstaculizan la creación de cursos nuevos. Asimismo, la brecha tecnológica del alumnado, o sea, acceso, uso y apropiación desigual de la tecnología. Si bien la academia se presenta como una estructura que dificulta el desarrollo hacia lo multimedia, la educación en periodismo, según la facultad, sigue enfrentando un reto mayor: el rezago del estudiantado en cuanto a las destrezas de redacción y de conocimiento general y cultural que formen a periodistas de excelencia. 


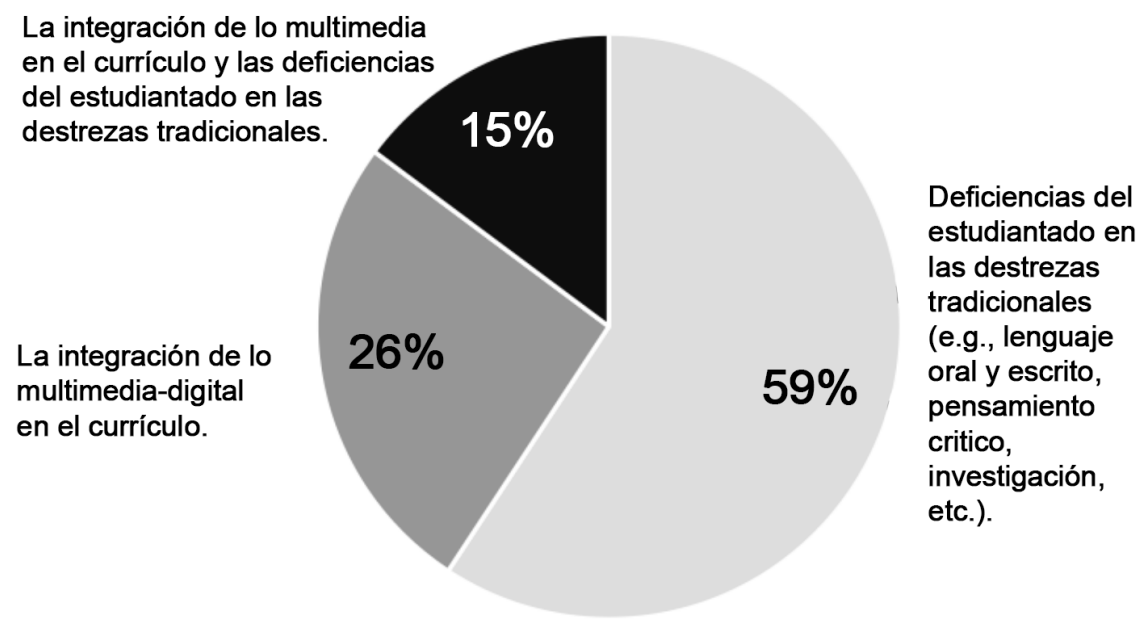

Figura 3. Retos que los docentes han enfrentado en la enseñanza durante los últimos diez años.

\section{Disposición hacia la tecnología}

El docente en Puerto Rico se siente cómodo con los procesos de enseñanza-aprendizaje del periodismo multimedia. Cerca de la mitad se percibe preparado para la cátedra de cursos teóricos y prácticos multimedia ( $55.55 \%$, cursos teóricos; $50 \%$ cursos prácticos); una tercera parte, bastante preparado (cursos teóricos, 33.33\%, y prácticos, 34.61\%), y una minoría, un poco preparado (cursos teóricos, $7 \%$, y prácticos, 15.38\%). (Véase fig. 4).

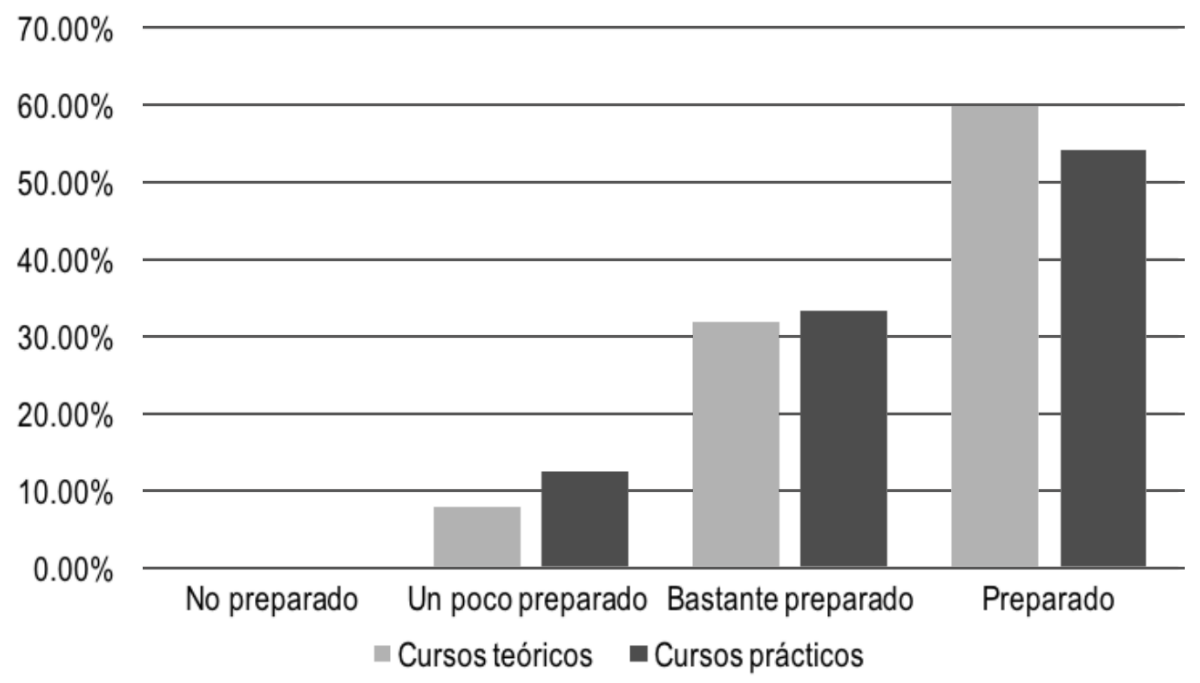

Figura 4. Percepción del profesorado de periodismo sobre su nivel de preparación para enseñar cursos multimedia teóricos y prácticos. 
No es de extrañar que, cuando se les preguntó, a los directores y al profesorado en Puerto Rico, cuán de acuerdo están con que la educación multimedia dificulta el proceso de enseñanza-aprendizaje, la mayoría de los encuestados está en desacuerdo con que obstaculiza tanto el proceso de enseñanza (directores, $87.50 \%$; profesores, $80 \%$ ) como el de aprendizaje (directores, $62.50 \%$; profesores, $86.67 \%$ ). Estos hallazgos son contrarios a los de Castañeda, Murphy y Heather (2005), quienes encontraron que tanto la facultad como el estudiantado en Estados Unidos percibían que el acercamiento multimedia ralentiza y diluye el proceso de enseñanza-aprendizaje. En otras palabras, ven como una dificultad la enseñanza y el aprendizaje que conduzca a pensar, reportar y escribir para el impreso, radio, televisión y ciberespacio de forma simultánea. Contrario a esa percepción, el estudio de Castañeda et al. (2005) halló que el estudiantado mostró una mejoría en las destrezas mencionadas.

La percepción positiva del profesorado en Puerto Rico hacia la educación multimedia conversa con las formas en que se los docentes se ven con respecto a la tecnología. El profesorado se definió como innovador (12\%), adaptador temprano (42\%) y parte de la mayoría temprana (46\%) en cuanto a su disposición hacia la tecnología. El primero se identifica por su tendencia a aventurarse hacia lo nuevo; el segundo, porque adopta la tecnología tan pronto se entera de sus bondades, y el tercero, por su disposición a acogerla cuando ciertos grupos sociales lo han hecho. Ninguno de los miembros de la facultad se definió como seguidor de la mayoría que ha adoptado la tecnología (mayoría tardía) o como quien la acoge cuando no tiene más remedio o, simplemente, no le interesa adoptarla (rezagado). El perfil del docente abre un espacio de posibilidad hacia la continua transformación de la academia en el país. Estudios han demostrado que aquellos profesores con mayor apertura a la tecnología exhiben mayores probabilidades de impulsar cambios curriculares hacia lo multimedia que aquellos con menor apertura (Coachie, 2008). (Véase fig. 5).

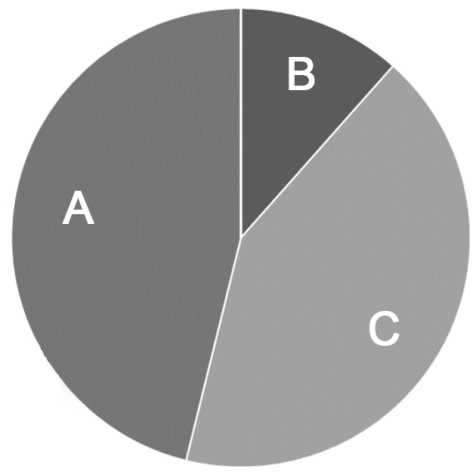

A Adopto la tecnología después de que se ha experimentado con ella y ciertos grupos sociales la han acogido y aceptado. $46 \%$

B Tan pronto se desarrolla la tecnología, me aventuro a experimentarla, sin saber si funciona o no. $12 \%$

C Tan pronto me entero de las bondades de la tecnología, soy de las primeras personas en adoptarla. $42 \%$

No me interesa adoptar ningún cambio tecnológico. 0\%

Adopto la tecnología cuando ya no me queda más remedio. $0 \%$

Cuando prácticamente la mayoría ya tiene la tecnología, la adopto. 0\%

Figura 5. Autopercepción del profesorado de periodismo sobre su disposición a la tecnología. 


\section{Educación continua del profesorado}

Los docentes en Puerto Rico han tomado cursos, talleres o seminarios sobre periodismo multimedia en los últimos diez años. El 71.43 por ciento de los directores afirmó que el profesorado de sus programas académicos ha participado en este tipo de actividad, mientras el 90 por ciento de la facultad encuestada dijo haber asistido a cursos de educación continua en los últimos diez años. De estos últimos, el 65 por ciento informó haber tomado entre dos y cinco talleres o seminarios; el 20 por ciento, entre seis y ocho, y el 15 por ciento, más de diez.

Ninguno de los departamentos le brinda apoyo económico total al profesorado para participar en cursos de educación continua, según los directores encuestados. No obstante, el 40 por ciento de los programas ofrece subvenciones para la mayoría, y el restante, para algunos de ellos. No es de extrañar, entonces, que el 44 por ciento de los docentes dijo no haber recibido ayuda financiera para cursos de educación continua. Mientras, el 28 por ciento recibió apoyo para algunos; el 20 por ciento, para la mayoría, y el 8 por ciento, para todos (véase fig. 6). Estos números contrastan con un estudio realizado en Estados Unidos, en que el 81 por ciento de la facultad y el 92 por ciento de los directores de los departamentos indicaron que sus unidades académicas apoyan económicamente los esfuerzos del profesorado para mantenerse al día en el campo (Oskam, 1996).

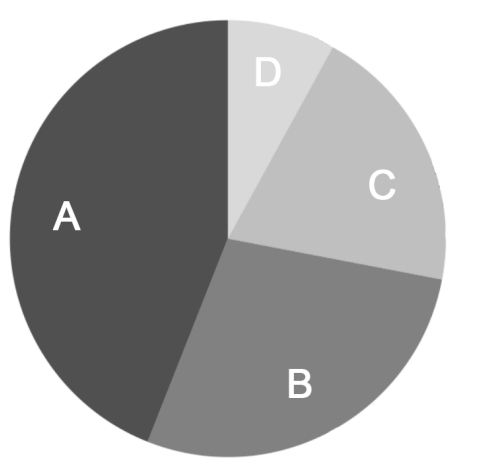
A No recibí subvención para ninguna de las actividades de capacitación $44 \%$
B Recibí subvención en algunos talleres o seminarrios $28 \%$
C Recibí subvención en la mayoría de los talleres o seminarios $20 \%$
D Recibí subvención en todos los talleres o seminarios $8 \%$

Figura 6. Nivel de apoyo institucional para asistir a talleres o seminarios de capacitación multimedia-digital según el profesorado de periodismo.

La limitada ayuda monetaria que se le otorga al docente posiblemente se relaciona tanto con las limitaciones económicas institucionales y del país - que sufre una marcada depresión desde 2008- como con la visión sobre en quién recae la responsabilidad de la educación continua. Ninguno de los directores o miembros de la facultad 
entiende que sea obligación de su departamento mantener al día a su profesorado. Los directores opinaron, en un 57.14 por ciento, que es tarea tanto del departamento como del docente, y el 42.86 por ciento, que es solo de la facultad. Los profesores, por su parte, entienden, en un 76.92 por ciento, que es un deber compartido del docente y el departamento, mientras un 23.08 por ciento considera que es solo de la facultad. Estos resultados se distancian de un estudio en Estados Unidos en el que el profesorado piensa, en un 79 por ciento, y los directores, en un 66 por ciento, que es responsabilidad exclusiva del docente mantenerse al día en su campo (Oskam, 1996).

\section{Perfil del profesorado: enseñanza y trasfondo profesional-académico}

El profesorado en Puerto Rico presenta un perfil profesional más que académico. Se compone de hombres (53.84\%) - aunque la encuesta fue contestada principalmente por mujeres (57.15\%) - de entre 40 y 59 años, que cuentan con una maestría o su equivalente como grado académico terminal (67.86\%), así como estudios en comunicación $(57.69 \%)^{5}$. (Véase tabla 2). Las mujeres representan la mayoría entre quienes tienen una maestría (58.88\%); no así entre quienes poseen un doctorado (hombres, $55.56 \%)$.

El docente de periodismo enseña cursos teóricos y prácticos $(82.85 \%)$ a nivel de pregrado $(86.11 \%)$, trabaja a tiempo completo $(61.54 \%)$ y no tiene plaza $(53.85 \%)$. (Véase tabla 2). La concentración de profesores que enseñan a nivel de pregrado se vincula con la proliferación de los programas en Comunicación con una orientación profesional. De los que dictan cursos prácticos, un 68.75 por ciento imparte los de contenido multimedia. Estos hallazgos van evidenciando la integración de este acercamiento en la formación académica del estudiantado.

El perfil profesional se exhibe, además, en la práctica periodística: sobre la mitad del profesorado cuenta con once años o más de experiencia en el campo (65.38\%), porcentaje que supera la académica (59.25\%). Asimismo, los docentes presentan una inclinación mayor hacia la creación profesional periodística sobre la investigación. De toda la facultad, un 45 por ciento dijo haber producido 41 o más trabajos periodísticos en los últimos diez años. Mientras, la mayoría no ha publicado, en los últimos diez años, artículos en revistas arbitradas especializadas (55\%), no especializadas (55.56\%) o no arbitradas (60\%), libros de texto (78.95\%) o investigativos (77.78\%), ni ha presentado propuestas de investigación para adquirir fondos externos $(68.42 \%)$. (Véanse tablas 3 y 4 ).

El instrumento presentaba la variable identidad de género, en la que el profesorado podía incorporar otra que no perteneciera a las categorías binarias hombre y mujer. Ninguno de los encuestados añadió otra categoría. 
Tabla 2. Datos demográficos del profesorado de periodismo por identidad de género.

\begin{tabular}{|c|c|c|c|}
\hline \multirow[t]{4}{*}{ Variables demográficas } & \multirow{2}{*}{$\frac{\text { Todos (as) }}{\%}$} & \multicolumn{2}{|c|}{ Identidad de género } \\
\hline & & Hombres \% & Mujeres \% \\
\hline & Población ( $N=39$ ) & 53,84 & 46,15 \\
\hline & Muestreo $(n=27)$ & 42,85 & 57,15 \\
\hline \multicolumn{4}{|l|}{ Edad } \\
\hline 30 años o menos & 7,41 & 0,0 & 14,29 \\
\hline $31-39$ & 18,52 & 25,0 & 14,29 \\
\hline $40-49$ & 29,63 & 25,0 & 28,57 \\
\hline $50-59$ & 25,93 & 33,33 & 21,43 \\
\hline 60 o más & 18,52 & 16,67 & 21,43 \\
\hline \multicolumn{4}{|l|}{ Grado terminal } \\
\hline Maestría o grado equivalente & 67,86 & 58,33 & 71,43 \\
\hline Doctorado & 32,14 & 41,67 & 28,57 \\
\hline \multicolumn{4}{|c|}{ Área de estudio del grado terminal } \\
\hline Comunicación & 57,69 & 58,33 & 57,15 \\
\hline Humanidades & 19,23 & 8,33 & 28,57 \\
\hline Educación & 11,54 & 16,67 & 7,14 \\
\hline Otras & 11,54 & 16,67 & 7,14 \\
\hline \multicolumn{4}{|l|}{ Con plaza o sin plaza } \\
\hline Con plaza & 46,15 & 50,0 & 42,86 \\
\hline Sin plaza & 53,85 & 50,0 & 57,14 \\
\hline \multicolumn{4}{|l|}{ Carga académica } \\
\hline A tiempo parcial & 38,46 & 25,0 & 50,0 \\
\hline A tiempo completo & 61,54 & 75,0 & 50,0 \\
\hline \multicolumn{4}{|l|}{ Tipo de curso que enseña } \\
\hline Teóricos y prácticos & 82,85 & 75 & 85,71 \\
\hline Prácticos & 14,29 & 16,67 & 14,29 \\
\hline Teóricos & 2,86 & 8,33 & 0,0 \\
\hline \multicolumn{4}{|l|}{ Nivel que enseña } \\
\hline Pregrado y posgrado & 11,11 & 16,67 & 7,14 \\
\hline Solo pregrado & 86,11 & 83,33 & 85,71 \\
\hline Solo posgrado & 2,78 & 0,0 & 7,14 \\
\hline
\end{tabular}


Tabla 3. Cultura académica del profesorado de periodismo.

\begin{tabular}{|c|c|c|c|}
\hline & Todos (as) \% & $\begin{array}{l}\text { Profesores (as) con maestría } \\
\text { como grado terminal } \%\end{array}$ & $\begin{array}{l}\text { Profesores (as) con doctorado } \\
\text { como grado terminal \% }\end{array}$ \\
\hline \multicolumn{4}{|c|}{ Años de experiencia docente } \\
\hline 0 años & 0,0 & 0,0 & 0,0 \\
\hline $1-5$ & 33,33 & 40,0 & 22,22 \\
\hline $6-10$ & 7,41 & 6,67 & 11,11 \\
\hline $11-15$ & 22,22 & 26,67 & 22,22 \\
\hline $16-20$ & 14,81 & 6,67 & 11,11 \\
\hline 210 más & 22,22 & 20,0 & 33,33 \\
\hline \multicolumn{4}{|c|}{ Artículos de comunicación en revistas arbitradas especializadas: últimos 10 años } \\
\hline 0 artículos & 55,0 & 69,23 & 28,56 \\
\hline $1-2$ & 20,0 & 23,08 & 14,29 \\
\hline $3-5$ & 10,0 & 0,0 & 28,57 \\
\hline $6-8$ & 10,0 & 7,69 & 14,29 \\
\hline $9-10$ & 0,0 & 0,0 & 0,0 \\
\hline $11-20$ & 5,0 & 0,0 & 14,29 \\
\hline \multicolumn{4}{|c|}{ Artículos de comunicación en revistas arbitradas no especializadas: últimos 10 años } \\
\hline 0 artículos & 55,56 & 72,72 & 28,57 \\
\hline $1-2$ & 27,78 & 18,18 & 42,86 \\
\hline $3-5$ & 0,0 & 0,0 & 0,0 \\
\hline $6-8$ & 11,11 & 9,09 & 14,28 \\
\hline $9-10$ & 0,0 & 0,0 & 0,0 \\
\hline $11-20$ & 5,55 & 0,0 & 14,28 \\
\hline \multicolumn{4}{|c|}{ Artículos de comunicación en revistas no arbitradas: últimos 10 años } \\
\hline 0 & 60,0 & 69,23 & 50,0 \\
\hline $1-2$ & 15,0 & 15,38 & 16,66 \\
\hline $3-5$ & 15,0 & 7,69 & 16,66 \\
\hline $6-8$ & 10,0 & 7,69 & 16,66 \\
\hline $9-10$ & 0,0 & 0,0 & 0,0 \\
\hline $11-20$ & 0,0 & 0,0 & 0,0 \\
\hline \multicolumn{4}{|c|}{ Libros de texto en comunicación: últimos 10 años } \\
\hline 0 libros & 78,95 & 76,92 & 83,33 \\
\hline $1-2$ & 21,05 & 23,08 & 16,66 \\
\hline \multicolumn{4}{|c|}{ Libros de investigación en comunicación: últimos 10 años } \\
\hline 0 libros & 77,78 & 84,62 & 66,67 \\
\hline $1-2$ & 22,22 & 15,38 & 33,33 \\
\hline \multicolumn{4}{|c|}{ Propuestas de investigación para adquirir fondos externos: últimos 10 años } \\
\hline 0 propuestas & 68,42 & 84,62 & 33,33 \\
\hline $1-2$ & 26,32 & 15,38 & 50,0 \\
\hline $3-4$ & 5,26 & 0,0 & 16,67 \\
\hline
\end{tabular}


No obstante, algunas diferencias por razón de género y nivel educativo se manifestaron en la experiencia docente y en los medios, así como en la producción académica y profesional, entre otras variables.

El profesorado con doctorado tiene más experiencia profesional y de enseñanza que aquel con maestría, así como mayor producción profesional e investigativa. Cuenta con un mínimo de seis años en la industria, mientras que el que tiene maestría puede llegar a la academia con menos de cinco años de experiencia profesional. El 77.77 por ciento de la facultad con doctorado posee once años o más de experiencia profesional frente a un 58.82 por ciento de los que tienen maestría (véase tabla 4). Asimismo, los hombres tienen más experiencia profesional que las mujeres: 75 por ciento de ellos posee más de 11 años versus 57 por ciento de ellas. En cuanto a la labor profesional, cuando se examina por nivel educativo, todos los que tienen doctorado han producido algún trabajo periodístico en los últimos diez años, mientras 8.33 por ciento de aquellos con maestría no han divulgado ninguno.

Tabla 4. Cultura profesional del profesorado de periodismo.

\begin{tabular}{|c|c|c|c|}
\hline & Todos (as) \% & $\begin{array}{l}\text { Profesores (as) con maestría } \\
\text { como grado terminal } \%\end{array}$ & $\begin{array}{l}\text { Profesores (as) con doctorado } \\
\text { como grado terminal \% }\end{array}$ \\
\hline \multicolumn{4}{|c|}{ Años de experiencia profesional } \\
\hline 0 años & 0,0 & 0,0 & 0,0 \\
\hline $1-5$ & 19,23 & 29,41 & 0,0 \\
\hline $6-10$ & 15,38 & 11,76 & 22,22 \\
\hline $11-15$ & 15,38 & 11,76 & 22,22 \\
\hline $16-20$ & 23,08 & 17,65 & 33,33 \\
\hline Más de 21 & 26,92 & 29,41 & 22,22 \\
\hline \multicolumn{4}{|c|}{ Trabajos periodísticos divulgados últimos 10 años } \\
\hline 0 trabajos & 5,0 & 8,33 & 0,0 \\
\hline $1-9$ & 20,0 & 25,0 & 12,5 \\
\hline $10-20$ & 25,0 & 8,33 & 50,0 \\
\hline $21-30$ & 5,0 & 8,33 & 0,0 \\
\hline $31-40$ & 0,0 & 0,0 & 0,0 \\
\hline Más de 41 & 45,0 & 50,0 & 37,5 \\
\hline
\end{tabular}

El docente con doctorado, además, muestra mayor experiencia en el salón de clases ante el que tiene maestría: un 77.77 por ciento cuenta con un mínimo de seis años, frente a un 60 por ciento que posee maestría o un grado equivalente (véase tabla 3). Asimismo, los hombres ostentan más experiencia en la enseñanza que las mujeres: 50 por ciento de los hombres tienen 16 años o más, frente a un 23.07 por ciento de las 
mujeres (véase gráfica 7). A este cuadro se le suma que una mayor proporción de las mujeres, frente a los hombres, labora a tiempo parcial y no cuenta con un puesto fijo en la institución.

Cuando se considera por nivel educativo, la mayor producción de artículos en revistas arbitradas se concentra entre aquellos que tienen doctorado. Sobre un 70 por ciento de los que ostentan el grado más alto han divulgado al menos un artículo en revistas arbitradas en los últimos diez años, y alrededor de un 66 por ciento han sometido propuestas de investigación para adquirir fondos externos (véase tabla 3). En ese sentido, el profesorado con doctorado, que suele estar compuesto por hombres, no solo cuenta con un acervo investigativo y profesional mayor, sino también exhibe mayor experiencia profesional y docente que el de maestría.

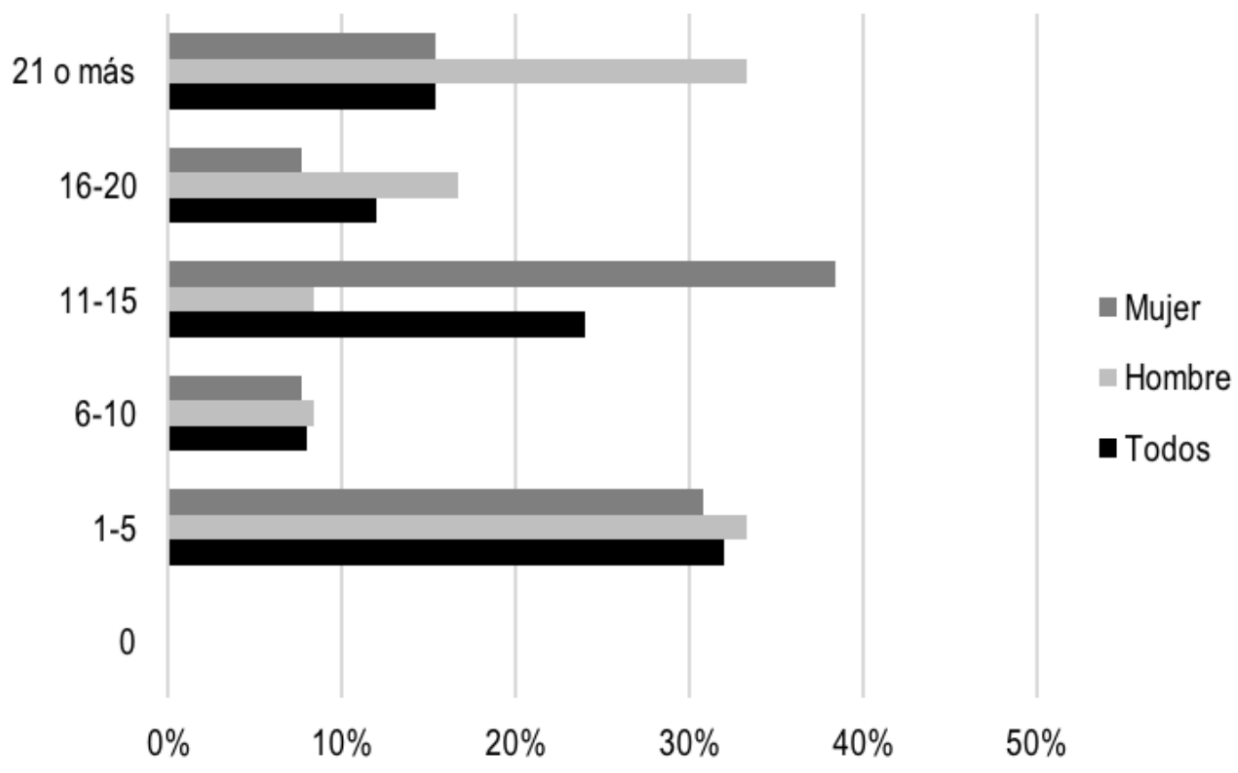

Figura 7. Años de experiencia docente del profesorado de periodismo por identidad de género.

\section{Conclusiones}

Los programas académicos que integran la enseñanza del periodismo en Puerto Rico han atemperado sus currículos al contenido multimedia-digital a base de un modelo multidisciplinario, y no interdisciplinario, en respuesta a las innovaciones del campo profesional. Las limitaciones que imponen las secuencias curriculares y la cantidad de horas crédito exigidas al estudiantado representan los principales obstáculos que, según los encuestados, impiden implementar con éxito los cambios multimedia en los programas educativos. Si bien las escuelas o departamentos han transformado muchos de los cursos, las prácticas emergentes más comunes, pero no mayoritarias, 
en la educación multimedia se reducen a la compra de equipo, lo que apunta a un nivel de transformación muy básico. Sin embargo, para muchos de los docentes, la falta de equipo y de instalaciones siguen señalándose como dificultades serias en el proceso de enseñanza-aprendizaje. Estos hallazgos se relacionan con los de un estudio entre las facultades de Comunicación en Latinoamérica que identificó la actualización permanente del equipo como uno de los obstáculos en la enseñanza multimedia (FELAFACS-UNESCO, 2009).

La facultad reconoce que los desafíos principales en la docencia en los últimos años se vinculan mayormente con la falta de dominio del estudiantado de las destrezas básicas de redacción, pensamiento crítico, investigación y lectura, más que con los cambios que ha traído el paradigma multimedia-digital. Estos resultados dialogan con estudios en diversos países, incluyendo Puerto Rico, en los que los medios y la academia identificaron, como muy importantes, las destrezas básicas en la formación del futuro periodista (Huang et al., 2006; Pierce \& Miller, 2007; Du \& Thornburg, 2011; Arroyo Cabello, 2011; Poynter Institute, 2013; García-Santamaría \& Barranquero Carretero, 2014; Lugo-Ortiz, 2016). Los desafíos señalados por el profesorado apuntan a una gran dificultad en el proceso de enseñanza-aprendizaje que obstaculiza, y prácticamente imposibilita, que los programas en periodismo en el país formen profesionales que puedan contribuir al debate público, por más que se intenten integrar los aspectos multimedia-digitales en los currículos. El conflicto se torna más complejo cuando apunta a unas deficiencias estructurales que desbordan la universidad, debido a que provienen de la educación primaria y secundaria.

Los docentes en Puerto Rico, pese a todo, se sienten cómodos con los cambios tecnológicos y entienden que la educación multimedia no dificulta el proceso de enseñanza-aprendizaje. Su apertura hacia la tecnología podría explicar las transformaciones de los programas y sentar las bases para que la educación interdisciplinaria cobre mayor peso en el futuro. No obstante, la enseñanza multidisciplinaria que se ha adoptado apunta hacia un nivel muy básico de innovación que no da cuenta de la producción periodística actual. Tal y como afirma Salaverría (2010), no es suficiente "mostrar cómo se ejerce el periodismo para internet, debemos enseñar a practicarlo con, en, sobre y mediante internet" (p. 248). La perspectiva interdisciplinaria provee el modelo para facilitar este tipo de enseñanza, por lo que las facultades en Puerto Rico deben considerar moverse hacia esa dirección.

La disposición de la facultad hacia la tecnología se une a otras características que apuntan a una cultura profesional en los centros educativos del país: el docente tiene maestría o su equivalente, estudios en comunicación, enseña cursos teóricos y prácticos, y tiene experiencia y producción profesional más que investigativa. Estos resul- 
tados conversan con la cultura profesional que se exhibe en Chile, pero con grados terminales más altos como en Estados Unidos, Alemania, entre otros países.

La facultad con doctorado en Puerto Rico exhibe mayor producción académica y experiencia profesional-docente sobre aquella con maestría o su equivalente. Estos resultados contradicen la noción popular, pero poco evidenciada, de que el profesorado con dicho grado terminal carece de experiencia en los medios. El docente con doctorado podría impulsar un modelo educativo con bases académicas y profesionales sólidas que fortalezcan los fundamentos de la profesión, la investigación, a la vez que robustece las destrezas profesionales. Sin embargo, este grupo constituye la minoría entre la facultad. Por lo tanto, atraer mayores profesores con dicho perfil representa una asignatura pendiente para las instituciones educativas, lo que se convierte en una dificultad seria cuando el país y, por lo tanto, sus instituciones públicas y privadas, enfrenta un periodo de depresión económica.

Esta investigación evidenció, además, unas diferencias por razón de género en las que las mujeres tienen menor nivel educativo y experiencia profesional que los hombres, así como ocupan más puestos sin plaza. En Puerto Rico, al igual que en otros países, las instituciones de educación superior que otorgan plazas suelen requerir un grado doctoral, educación que, igualmente, facilita que el candidato adquiera un puesto a tiempo completo. Los datos revelados en este estudio ameritan tomarse como punto de partida para generar investigaciones que exploren cómo las estructuras sociales - los roles de género y las asignaciones culturales que estos imponen, entre otros- afectan la labor de las profesoras universitarias. Explorar con mayor detenimiento estas tendencias cobra mayor importancia cuando las mujeres representan la mayoría del estudiantado en la universidad pública del país desde la década de los sesenta, aunque no la mayoría entre los que cuentan con un doctorado para enseñar periodismo. Indagar este tema no solo aquilataría la repercusión que tiene el género en la desigualdad de acceso a mayor conocimiento teórico-investigativo - que abre la puerta a publicar y ocupar posiciones permanentes dentro de los departamentos en los que enseñansino en alcanzar mayor independencia y seguridad económica.

El perfil profesional de la facultad en Puerto Rico explica la disposición de los programas para integrar lo multimedia en los currículos, mas no ha demostrado ser suficientemente fuerte para fundar una cultura profesional o académica sólida. Como establece Mellado (2011) al referirse a los docentes en Chile, el perfil profesional de la facultad puede contribuir a la profesionalización de la enseñanza del periodismo en términos prácticos, pero no al desarrollo del nuevo conocimiento en una tradición académico-universitaria.

La educación continua ha sido un vehículo que ha ayudado a actualizar al docente en Puerto Rico, y debe serlo cuando se cuenta con una facultad que, en ocasiones, no 
tiene producción creativa ni investigativa, particularmente la que posee maestría. Sin embargo, el profesorado entiende que el adiestramiento es una responsabilidad compartida entre la academia y el docente. El problema con esta visión es que, en la medida que la institución no provea subvenciones para la capacitación, el docente podría desentenderse de esa tarea.

Si bien los programas que enseñan periodismo se han ido transformando, el profesorado debe fortalecer su perfil mediante la educación continua y la producción académica y profesional. Solo robusteciendo la cultura profesional y académica —ambas, no una o la otra-, las universidades podrán innovar los currículos, contribuir a la profesión, crear conocimiento y, con ello, acometer el reto que presenta el campo en tiempos multimedia.

\section{Referencias}

ACEJMC (2016). Public Accountability and Disclosure. Accrediting Council on Education in Journalism and Mass Communications. En Área de Evaluación, Planificación, Estadísticas e Investigación. Recuperado de https://www2.kuedu/ acejmc/STUDENT/ Public_AccountabilitySHTML\#INT.

Arroyo Cabello, M. (2011). Aproximación al perfil del periodista en la posmodernidad. Razón y Palabra 76, mayo-julio. Recuperado de http://wwwrazonypalabra.org.mx/N/ N76/varia/2a\%20 entrega/25_Arroyo_V76.pdf.

Auman,A.\&Lillie,J.(2008). An Evaluation of Team-teaching Models in a Media Convergence Curriculum. Journalism and Mass Communication Educator, 62(4), 360-375.

Boers, R., Ercan, E., Rinsdorf, L. \& Vaagan, R.W. (2012). From Convergence to Connectivism: Teaching Journalism 2.0. Online Journal of Communication and Media Technologies, 2 (4), octubre, 52-64.

Bronstein, C. \& Vaughn, S. (1998). Willard G. Bleyer and the Relevance of Journalism Education. Journalism and Mass Communication Monographs, 166.

Carnegie-Knight Initiative on the Future of Journalism (2011). Joan Shorenstein Center for Press, Politics, and Public Policy. Cambridge: Harvard University.

Castañeda, L., Murphy, H. \& Heather, J. (2005). Teaching Print, Broadcast, and Online Journalism Concurrently: A Case Study Assessing a Convergence Curriculum. Journalism \& Mass Communication Educator, 60(1), 57-70.

Coachie,M.A.(2008). Convergencein the Curriculum:A Survey of CollegeCommunications Programs. (Tesis de maestría en Comunicación Masiva). University of Florida, Gainesville, Florida.

Dates, J. L. (2006). Rethinking Journalism Education. Journalism Studies, 7 (1), 144-146.

De Mendonça, T., Henrique Pereira, F. \& Leal-Adghirni, Z. (2012). Formación y producción periodística en internet: desafíos y perspectivas en el escenario brasileño. Palabra Clave, 15 (1), abril, 26-53. 
Deuze, M. (2004). What is Multimedia Journalism? Journalism Studies, 5 (2), 139-152.

Deuze, M. (2006). Global Journalism Education: A conceptual approach. Journalism Studies, $7(1), 19-34$.

Deuze, M., Neuberger, C. \& Paulussen, S. (2004). Journalism Education and Online Journalists: Belgium, Germany, and The Netherlands. Journalism Studies (5)1, 19-29.

Dickson, T. (2000). Mass Media Education in Transition: Preparing for the 21st. Century. Nueva Jersey: Lawrence Erlbaum Associates, Publishers.

Dickson, T. \& Brandon, W. (2000a). Media Criticisms of US Journalism Education: Unwarranted, Contradictory. Asia Pacific Media Educator, 11 (8), 42-58.

Dickson, T. \& Brandon, W. (2000b). The Gap Between Educators and Professional Journalists. Journalism \& Mass Communication Educator, 55(3),50-67.

Donsbach, W. \& Fiedler, T. (2008). Journalism School Curriculum Enrichment: a Midterm Report of the Carnegie-Knight Initiative on the Future of Journalism Education. Cambridge, USA: Joan Shorenstein Center on the Press, Politics and Public Policy, John F. Kennedy School of Government, Harvard University.

Du, Y. R. \& Thornburg, R. (2011). The Gap Between Online Journalism Education and Practice: The Twin Surveys. Journalism and Mass Communication Educator, 66(3), 217-230.

EscalanteRengifo,G., García de Jesús, Y. \& Oropeza Herrera,F. (2010).Los estudios graduados en comunicación en Puerto Rico: institucionalización y producción académica. Revista Latinoamericana de Ciencias de la Comunicación, 7 (13), julio-diciembre, 80-89.

Escuela de Comunicación (s. f.). Historia de la Escuela. Universidad de Puerto Rico. Recuperado de http://copu.uprrp.edu/mision.html.

ESJ (s. f.). École Supériore de Journalisme. Recuperado de http://www.esj-paris.com/Site/8histoire-de-l-esj-paris.html.

Fedler, F. \& Counts, T. (1982). National J-faculty survey reveals job likes, dislikes. Journalism Educator, 37(3), 3-6.

Fedler, F., Counts, T. \& Smith, R. (1984). Survey compares attitudes of male, female professors. Journalism Educator, 39(3), 3-8.

FELAFACS-UNESCO (2009). Mapa de los centros y programas de formación en Comunicación y periodismo en América Latina y el Caribe, Lima, UNESCO.

Figueiredo Filho, D. B., Paranhos, R., Rocha, E. C. D., Batista, M., Silva Jr., J. A. D., Santos, M. L.W. D., \& Marino, J. G. (2013). When is statistical significance not significant? Brazilian Political Science Review, 7 (1), 31-55.

Fröhlic, R. \& Holtz-Bacha, C. (2003). Journalism Education in Europe and North America: An International Comparison. Nesskill, Nueva Jersey: Hampton Press.

Fuentes Navarro, R.(1992).El estudio de la comunicación desde una perspectiva sociocultural en América Latina. Diálogos de la Comunicación, 32. Recuperado de http://hdl.handle. net/11117/2854. 
García-Santamaría, J. V. \& Barranquero Carretero, A. (2014). La formación universitaria en periodismo. ¿Mera capacitación tecnológica o una formación integral en el nuevo ecosistema informativo? Historia y Comunicación Social, 19, enero, 641-651.

Grisales, J. M. (2012). Enseñar periodismo para leer y narrar la sociedad del siglo XXI. Cuadernos de Información, 30, enero-junio, 111-122.

Hansen, K.A. (2005).Values and Competencies from the Clash of Professional and Academic Cultures. Journalism and Mass Communication Educator, 60(2), 130-134.

Hedges, E. (2006). First School of Journalism Turns 100. Newseum. Recuperado de http:// www.newseum.org/news/2008/09/first-school-of-journalism-turns-100.html.

Hirst, M. (2010). Journalism Education “Down Under": A Tale of Two Paradigms. Journalism Studies, 11 (1), 83-98.

Huang, E., Davison, K., Shreve, S., Davis, T., Bettendorf, E. \& Nair, A. (2006). Bridging Newsrooms and Classrooms: Preparing the Next Generation of Journalists for Converged Media. Journalism and Communication Monographs.

King, E. (2008). The Role of Journalism History, and the Academy, in the Development of Core Knowledge in Journalism Education. Journalism \& Mass Communication Educator, 63(2), 166-178.

Koivisto, J. \& Thomas, P. (2007). Mapping Communication and Media Research: Germany. Helsinki: Communication Research Center, University of Helsinki.

Kraeplin, C. \& Criado, C.A. (2005). Building a Case for Convergence Journalism Curriculum. Journalism and Mass Communication Educator, 37(1), 47-56.

López García, X. (2010). La formación de los periodistas en el siglo XXI en Brasil, España, Portugal y Puerto Rico. Revista Latina de Comunicación Social, 65, 231-243.

Lowrey, W., Daniels, G. \& Becker, L. (2005). Predictors of Convergence Curricula on Journalism and Mass Communication Programs. Journalism and Mass Communication Educator, primavera, 32-46.

Lugo-Ortiz, L. (2016). La academia, los medios y el profesional ideal: el periodista generalista multimedia. Communication \& Society, 29 (4), 271-286.

Mellado, C. (2011). Examining Professional and Academic Culture in ChileanJournalism and Mass Communication Education. Journalism Studies, 12 (3), 375-391.

Morton, L. \& Beard,F.(2005). Faculty Productivity and Carnegie Institutional Characteristics within AEJMC Programs. Journalism \& Mass Communication Educator. 60 (2), 176-189.

Nolan, D. (2008). Journalism, Education and the Formation of 'Public Subjects'. Journalism, 9, 733-949.

Oskam, J.(1996). How Educators Keep Up-to-Date with the Changes in the Field. Journalism \& Mass Communication Educator, 51 (3), 45-53.

Pierce, T. \& Miller, T. (2007). Basic Journalism Skills Remain Important in Hiring. Newspaper Research Journal, 28 (4), otoño, 51-61. 
Poynter Institute (2013). Poynter Releases Results of 2013 Study on the Future of Journalism Education, comunicado de prensa. Recuperado de http://about.poynter.org/about-us/ press-room/poynter-announces-results-2013-study-future-journalism-education.

Rahkonen, J. (2007). Mapping Media and Communication Research: Australia. Research report, Helsinki: Communication Research Centre, University of Helsinki.

Reese, S. D. (1999). The Progressive Potential of Journalism Education: Recasting the Academic versus Professional Debate. The International Journal of Press/Politics, 4 (4), 70-94.

Royal, C. (2005). Teaching web design in journalism and mass communications programs: Integration, judgment, and perspective. Journalism \& Mass Communication Educator, 59(4), 399-414.

Sahin, I. (2006) Detailed Review of Rogers' Diffusion of Innovations Theory and Educational Technology-Related Studies Based on Rogers' Theory. The Turkish Online Journal of Educational Technology, 5, 14-23.

Salaverría, R. (2003). Retos pendientes en la formación de periodistas para la era digital. Profesionales para un futuro globalizado. Actas del XVII Congreso Internacional de Comunicación. Pamplona: Ediciones Eunate, 387-397.

Salaverría, R. (2010). ¿Ciberperiodismo sin periodistas? Diez ideas para la regeneración de los profesionales de los medios digitales. En Campos Freire (coord.).El cambio mediático. Zarmora/Sevilla: Comunicación Social, 236-249.

Salaverría, R. (2011). Online Journalism Meets the University: Ideas for Teaching and Research. Brazilian Journalism Research, 7 (11), 137-152.

School of Journalism (1987). Planning for Curricular Change in Journalism Education: Project on the Future of Journalism and Mass Communication Education. Eugene: Oregon University.

Singer, J. B., Craig, D., Allen, C., Whitehouse, V., Dimitrova, A. \& Sanders, K. (1996). Attitudes of Professors and Students About New Media Technology. Journalism and Mass Communication Educator, 51(2), 36-45.

Stone, G. C. (1982). Professional Experience Gap Between PhDs, non-PhDs Narrows, "Roundtable" discloses. Journalism Educator, 37(1), 3-5.

Tejedor Calvo, S. (2006). La enseñanza del ciberperiodismo: hacia una transversalidad mixta.Zer, 21, 219-239.

Weaver, D. \& Wilhoit, C. (1988). A Profile of JMC Educators: Traits, Attitudes and Values. Journalism Educator, 43 (2), 4-41. 